\title{
The effect of incentives in web surveys: application and ethical considerations
}

\author{
Cihan Cobanoglu \\ University of Delaware \\ Nesrin Cobanoglu \\ University of Baskent
}

\begin{abstract}
Although researchers use internet-based surveys more often than ever in their research, there is little research on the effect of incentives on response rate, speed and cost. This study attempts to fill in some of the blanks by comparing the different incentives offered to respondents of web-based surveys. The results indicate that offering a luggage tag to each respondent and including them in a draw for a bigger value prize (a personal digital assistant) yields the highest response rate. In terms of response speed, there are no significant differences among each incentive group. The most expensive group in terms of costs was the combination of luggage tag and prize draw.
\end{abstract}

\section{Introduction}

As the number of internet users in the world doubles every year, reaching nearly 500 million in 2001 (Internet User Numbers 2002), researchers are using internet tools such as email and web-based surveys more and more as methods of data collection. According to Dillman (1999), the most significant advances in the survey methodology during the twentieth century were the introduction of random sampling in the 1940s and telephone interviewing in the 1970s. However, researchers today are witnessing similar, if not bigger, significant advances in the field of survey methodology with the introduction of technology-based surveys.

Today, mail and telephone surveys have reached maturity (Dillman 1999). However, there is not much research focusing on technology-based survey methodologies, simply because they have only been introduced in the last decade. The analysis of past research on the use of the internet as a survey medium has shown that there is a wide variation in response rates, speed of response and response quality (Sheehan \& McMillan 1999; Cobanoglu et al. 2001). Since the number of studies using these 
methodologies is limited and the populations and research topics they use are different, they may not give researchers a clear idea on the effects of the internet-based surveys.

The purpose of this study is to explore the effects of incentives on response rate, speed, and cost in online surveys. An experimental study was designed to measure the effect of different incentive options in online surveys.

\section{Review of literature}

Surveys are one of the most popular data collection methods for academic research in a variety of fields, such as business, marketing, economics, psychology and hospitality (Roztocki 2001). Although internet-based surveys are used extensively by researchers as a data collection method, there has been relatively limited published research on the specifics of this methodology (Zhang 2000; Cobanoglu et al. 2001; Roztocki 2001). Consequently, researchers intending to explore the potential of internetbased surveys for primary data collection are left with limited guidance (Roztocki 2001). This study focuses on the use of incentives in web-based surveys.

\section{Why do people respond to surveys?}

Albaum et al. (1998) suggested four theoretical perspectives that have been proposed as being applicable to the survey response decision process: social exchange, cognitive dissonance, self-perception and commitment/ involvement. Social exchange subsumes the features of self-perception, cognitive dissonance, and commitment/ involvement (Helgeson et al. 2002). Social exchange theory suggests that participation in an exchange is determined by value assessments based on cost-benefit analysis made by the parties of the exchange (Dillman 1978). Groves and Couper (1998) described these cost-benefit assessments as a bookkeeping system of debits and credits rooted in an individual's self-perception and commitment/ involvement with the object. Cognitive dissonance suggests that individuals will experience discordant feelings if their behaviour is not consistent with pre-existing attitudes. Self-perception refers to the idea that individuals are more likely to engage in behaviour when that behaviour is consistent with their view of themselves (Helgeson et al. 2002). Finally, commitment/involvement contends that an individual who is strongly committed to a behaviour or activity will be unlikely to terminate that 


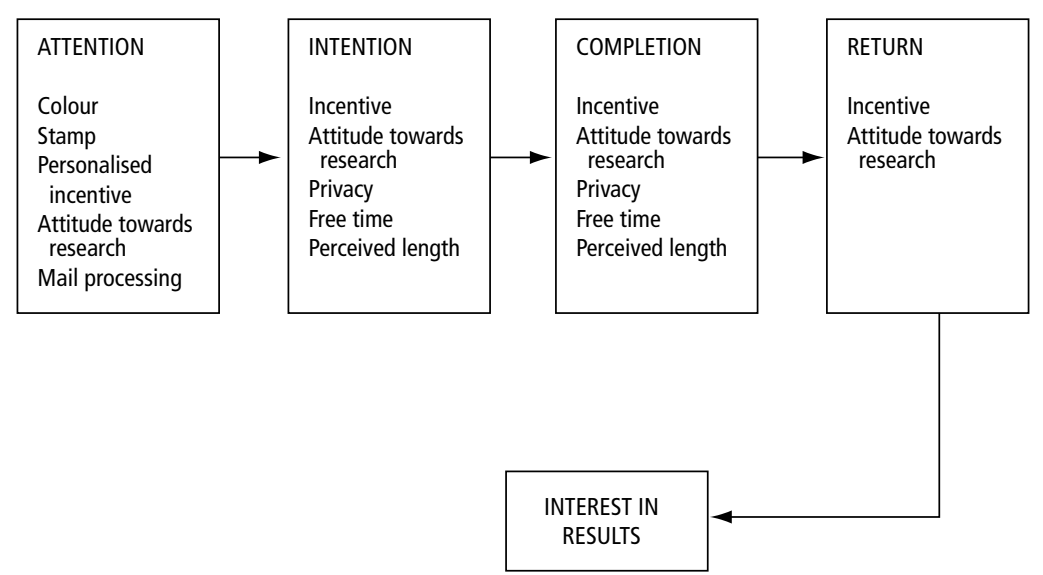

Source: Adapted from Helgeson et al. (2002)

Figure 1 Survey response hierarchy of effects model

behaviour or activity. Cobanoglu et al. (2001) suggest that use of incentives in surveys contributes mostly to the cost-benefit assessment by providing a solid benefit to the respondent. Helgeson et al. (2002) describe the response process in Figure 1. Incentive is a determinant in each level of the model.

\section{Web-based surveys}

Researchers have started to use internet-based surveys along with other media such as mail, telephone and fax (Cobanoglu et al. 2001). Table 1 shows the specifications of web-based surveys. The biggest advantages of web-based surveys compared with traditional mail and fax surveys are

Table 1 Specifications of web-based surveys

\begin{tabular}{ll}
\hline Factor & Web-based \\
\hline Coverage & Low \\
Speed & High \\
Return cost* & No cost to the respondent \\
Incentives & Coupons may be included \\
Wrong addresses & High \\
Labour needed & Low \\
Expertise to construct & High \\
Variable cost/each survey & No cost \\
\hline
\end{tabular}

* In the US. In some other parts of the world, there may be a very small fee for returning the survey 
efficiency, speed and low cost (Cobanoglu et al. 2001; Roztocki 2001). The use of web-based surveys eliminates mailing costs for questionnaires, reduces the cost of coding respondents' data, completes the data collection process faster (Cobanoglu et al. 2001), reduces human error and reaches respondents in different geographic areas effectively (Roztocki 2001). Even though there may be some potential problems with web-based surveys, such as multiple responses from the same participant, method bias and responses from unintended respondents, the current survey software packages such as Perseus Surveys prevent most of these problems.

One problem that may not be prevented by the use of sophisticated webbased survey software is method bias. If a researcher only uses web-based methods when surveying a sample where not all members of the population have access to the internet, the results may be biased. However, Cobanoglu et al. (2001) suggested that using a 'uniform mixed methodology' would help prevent this bias. Uniform mixed methodology refers to use of the internet-based method for the parts of the sample that have internet access, while for the other members of the sample who do not have internet access, other accessible methods should be used, such as mail or fax. However, if a uniform survey design is not achieved, then additional bias may exist. In other words, the major potential problem with using mixed-mode surveys for the same population is defined as the measurement differences between modes (de Leeuw 1992; Schwarz et al. 1992; Dillman 1999). This difference may even result in different analytical conclusions and recommendations.

Dillman (1999) suggested that there are four main reasons for differences between different modes: social desirability, acquiescence, question order effects, and primary/recency effects. This potential problem may be prevented, if not completely eliminated, by applying a uni-mode design which focuses on writing and presenting questions in a way that ensures respondents receive a common mental stimulus, i.e. whatever format the respondent sees the questionnaire in, he/she will understand it in the same way as the other respondents. However, most of the research on mixed-mode surveys has focused on the differences between mail and telephone, mail and face-to-face interview, and telephone and face-to-face interview. There is little research on the measurement differences between mail-, fax-, and internet-based surveys. Different from mixed-mode designs that employ telephone and mail methods only, the survey data obtained by using mail-, fax- and internet-based surveys may not be statistically different, provided that the surveys do not depend on printing quality, complex graphics and colour. 


\section{Web survey preparation}

There are three different methods for designing web-based surveys. The first method is using web publishing software such as Microsoft FrontPage or Macromedia Dreamweaver. Even though preparing a web-based survey with web publishing software is easy and takes little time, the biggest disadvantage is that designing multiple-page surveys is extremely difficult, so the researchers are limited to single-page surveys. Additionally, advanced features such as branching, i.e. preventing the same user from responding more than once, are not easily incorporated. The second method is using web-based survey design software such as Perseus. The survey may be imported from a text processor format such as Microsoft Word into the program. Advanced features such as multiple pages, branching, and respondent access control are a snapshot in this software. The disadvantage of using this software is that for advanced features the researcher has to provide the server with details of where the survey and the database will be hosted. This will create some complications, which an average user may not be able to handle easily. The third option for designing web-based surveys is to use web-based survey design sites such as Zoomerang.com. Even though these websites usually offer this service free of change, they may place a banner on the survey website, which may distract respondents and therefore create bias. Also, the features these sites offer are usually very simple.

\section{Use of incentives in surveys}

There is substantial evidence that responses to mail questionnaires are enhanced through the provision of monetary incentives (Huck \& Gleason 1974; Armstrong 1975; Church 1993). Studies have also explored whether incentives are more effective when they are larger or when they are paid in advance of participation (Shank et al. 1990; Collins et al. 2000). In general, they find that prepayment increases responses. Increasing the size of incentives also enhances response rate, but again only when the incentives (large or small) are paid in advance of participation (Church 1993).

Shank et al. (1990) have investigated the effect of cash versus non-cash incentives in mail surveys. Non-cash (mini-calculator) and cash (50 cents) incentives to every person who completed the survey increased the response rate significantly in a foodservice sample. In addition, the perceived cost of the non-cash incentives is three times greater than the actual cost of the incentive. Finally, Shank et al. (1990) demonstrated the 
effectiveness of immediate rather than delayed cash incentives. There were, however, no significant differences between the immediate and delayed non-cash incentives.

Another study assessed the effect of incentive size on response rates, data quality and cost in a digestive health status mail survey of a community sample of people enrolled on a health plan (Shaw et al. 2001). Results indicated that a US\$5 incentive resulted in a higher response rate among a community patient sample than a US $\$ 2$ incentive. However, the response rates in the $\$ 2$ example approached the level of the $\$ 5$ incentive and costs were significantly lower when the full follow-up protocol was completed. Response rates were marginally increased by follow-up phone calls.

Researchers have used different versions of non-cash incentives. Vandermeer (2000) suggested that using a telephone card as an incentive had positive effect on response rates. Brennan (1992) suggested that a 50 cent incentive sent with the first mail-out increased the response rate significantly. Similarly, another health care survey study found that financial incentives increased response rates significantly (Wang et al. 2002). In terms of the amount of the cash incentive, Halpern et al. (2002) suggested that a $\$ 10$ incentive yielded significantly more responses than a $\$ 5$ cash incentive in a health care survey. Helgeson et al. (2002) reported that a monetary incentive has a significant effect on response rates.

\section{Ethical considerations}

The field of ethics, also called moral philosophy, involves systematising, defending and recommending concepts of right and wrong behaviour (Cobanoglu 1998). In other words, it is the philosophy of values. In the past, the philosophy of values investigated the issues that affect a small society and incidents. However, with the increasing use of technology, the need for ethics increased (Cobanoglu \& Unsal 1998). In this paper, the authors discuss the ethical considerations regarding the use of incentives in web surveys.

\section{The need for this study}

Although there is extensive research on the effects of incentives in mail surveys, there is limited research on the effects of incentives in online surveys. The purpose of this study is to explore the effects of incentives on response rate, speed and cost in online surveys. 


\section{Methodology}

The survey was distributed to 1200 randomly chosen managers from American Management Association (AMA) members listed in the organisation's directory. In cases where the randomly chosen member did not have an email address, the next record was selected until a record with an email address was found. Three hundred managers were randomly assigned to one of the four incentive method groups: luggage tag (LT), prize draw for a personal digital assistant (PDA), both prize draw and luggage tag (PDA + LT), and control. These incentives were selected because of their use in previous research (Cobanoglu et al. 2001; Ray et al. 2001). Ray et al. (2001) found that in $57 \%$ of the web-based surveys conducted, respondents were promised survey results as an incentive to participate. In about $36 \%$ of the surveys, respondents were promised inclusion in a draw/raffle to encourage participation. And in $7 \%$ of the surveys they were given a small token for participating. Cobanoglu et al. (2001) offered a luggage tag to every respondent who completed the questionnaire fully and obtained a $23.4 \%$ response rate. Therefore, in this research a luggage tag was offered as an incentive to respondents. In addition, the low cost of offering a luggage tag was a factor in selecting it as an incentive for research. Similarly, other researchers offered a draw/raffle for a bigger value incentive, such as a personal digital assistant (PDA) or plane ticket to a specified destination (Ray et al. 2001).

A combination of incentives is usually not used by researchers. One of the objectives of this research was to investigate the combined effect. A control group was also created to measure the response rate and speed without an incentive. Clearly, there are many other incentives that can be used in online research. Some other examples may include vouchers for online merchants (i.e. Amazon.com gift certificate), discount coupons for online merchants (i.e. 10\% off at Barnes and Noble), free or discounted subscriptions to online publications (i.e. one month's free access to the Wall Street Journal Online), or a contribution to a charity on behalf of the respondent. In order to encourage the managers to respond, the survey topic covered a subject of interest to them: online travel patterns. A covering letter introducing the survey was personally addressed to each manager. Each manager received the same introduction with different incentive offerings depending upon which group they were in. Detailed records of each step of the procedure were kept in order to facilitate analysis. This included the dates the messages were sent, the return times and dates, and the costs. 
An email invitation message was sent to the managers along with a covering letter and the website address (URL) for the survey. A unique website address was created for each respondent with the help of a common gateway interface (CGI) protocol. Thus, the responses were tracked and the possibility of a response from a non-sample source eliminated. The internet protocol (IP) numbers were obtained along with the time and date the survey was completed by the respondent. Also, another CGI script was used to eliminate multiple entries by the same person. The invitation emails were sent using Microsoft Word and Outlook Express 5 by using the 'mail merge' feature.

\section{Dependent variables}

There were two primary dependent variables in this study:

1. Adjusted response rate. This rate was calculated by dividing the net responses by the net email invitations sent to the respondents.

2. Response speed. All emails were sent at the same time using four computers. The response speed was recorded for each method and the average response speed was calculated.

Individuals not responding to a survey or those responding to a survey because of special interests represent a potential bias (Cahalan 1951; Adams 1956). In order to prevent the 'special interest' bias, the authors chose a sample of managers that consisted of a sufficiently large group yet focused on an area that was likely to be of interest to every travelling manager, namely online travel patterns.

\section{Findings}

All email invitations were sent on the same day. Of the original 1200 surveys, 194 emails came back as undelivered. This may be due to the fact that managers change workplace along with their email addresses and they do not update their contact information with AMA (see Table 2).

\section{Response rate}

The chi-square test was conducted to determine if there was an overall significant difference across the different incentive groups. The chi-square 
Table 2 Response rate and speed comparison of different incentive groups

\begin{tabular}{lccccc}
\hline & Luggage tag & PDA draw & $\begin{array}{c}\text { Both luggage tag } \\
\text { and PDA }\end{array}$ & Control group & Total \\
\hline Sample size & 300 & 300 & 300 & 300 & 1200 \\
Number not deliverable & 49 & 52 & 39 & 54 & 194 \\
Percentage not deliverable $^{1}$ & 16.3 & 17.3 & 13.0 & 18.0 & 16.2 \\
Effective sample size $^{2}$ & 251 & 248 & 261 & 246 & 1006 \\
Surveys completed & 79 & 51 & 109 & 59 & 298 \\
Raw response rate (\%) $^{3}$ & 26.3 & 17.0 & 36.3 & 19.6 & 24.8 \\
${\text { Adjusted response rate }(\%)^{4}}^{4}$ & 31.4 & 20.5 & 41.7 & 23.9 & 29.6 \\
Days taken to respond & 5.1 & 4.9 & 3.9 & 5.7 & 4.9 \\
\hline
\end{tabular}

Notes:

1: Number not deliverable/sample size

2: Sample size/number not deliverable

3: Surveys returned/sample size

4: Surveys returned/effective sample size

value was 17.208 , which was significant at the 0.001 level with three degrees of freedom.

The average response rate was $29.6 \%$, with the luggage tag method being $31.4 \%$, PDA prize-draw method being $20.5 \%$, the combined incentive method being $41.7 \%$, and control group with no incentive being $23.9 \%$ (Table 2). Overall, using the arc sine square root transformation ANOVA (Kempthorne 1952), there was a significant difference among the response rates for all methods (chi-square $=17.208, \mathrm{df}=3, p=0.001$ ).

Table 3 shows the pair comparisons between different incentive methods. An LSD-type $z$-test shows significant differences in response rates between luggage tag and prize-draw methods (chi-square $=4.532$, $\mathrm{df}=1, p=0.033$ ) and between prize draw and LT + prize draw (chi-square $=13.979, \mathrm{df}=1, p=0.000)$, and between $\mathrm{LT}+$ prize draw and control groups (chi-square $=9.15, \mathrm{df}=1, p=0.002$ ) .

Table 3 Post hoc pair comparisons between different incentive methods

\begin{tabular}{lccccccc}
\hline & \multicolumn{2}{c}{ Prize draw } & \multicolumn{2}{c}{ LT + prize draw } & \multicolumn{2}{c}{ Control } \\
\cline { 2 - 8 } & LT $^{*}$ & Chi-sq & $p$ & Chi-sq & $p$ & Chi-sq & $p$ \\
\hline LT & 4.532 & 0.033 & 2.706 & 0.100 & 1.968 & 0.161 \\
Drawing & & & 13.979 & 0.000 & 0.530 & 0.466 \\
LT + drawing & & & & & & 9.150 & 0.002 \\
Control & & & & & & & \\
\hline
\end{tabular}

*Luggage tag 


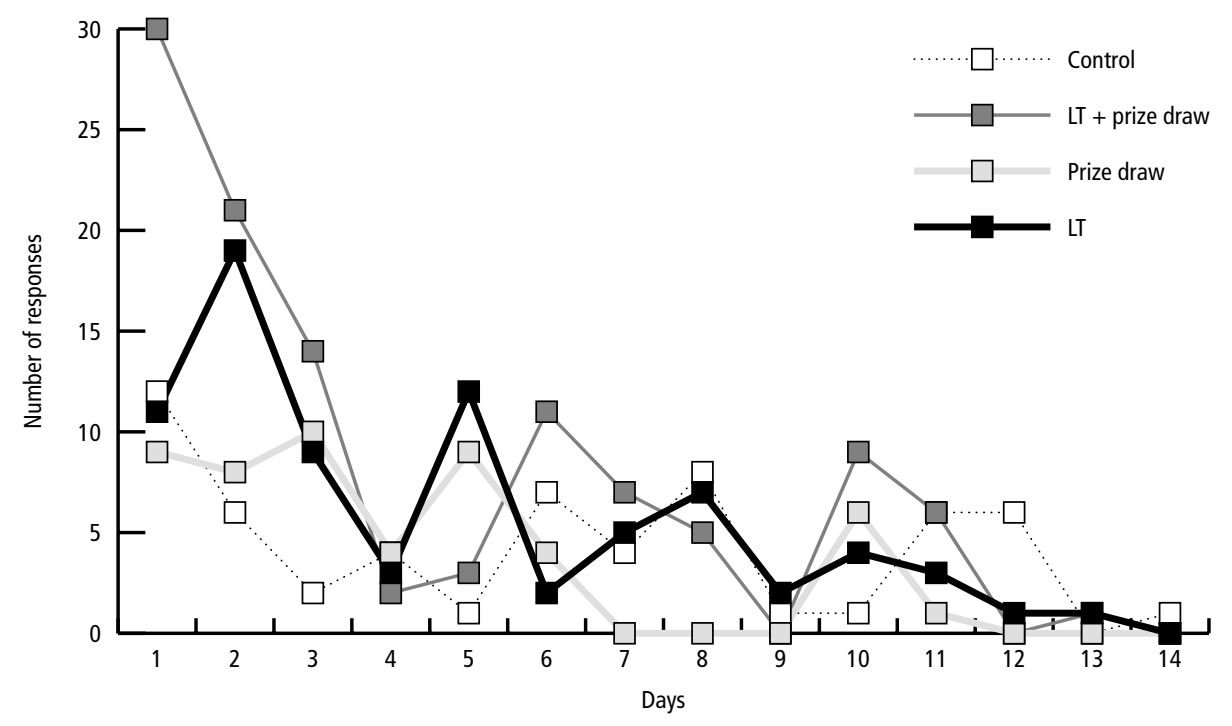

Figure 2 Response return patterns for each group

\section{Response speed}

Figure 2 shows the response return patterns for each group. Overall, there was no significant difference among four groups in response speed. As can be seen from Figure 2, the LT + prize draw group received the most responses on the first day of the survey distribution process. In each method there was a jump after the 5th-day reminder and 10th-day reminder.

\section{Costs}

Table 4 shows the fixed and variable costs for the luggage tag (LT), prize draw for PDA (prize draw), luggage tag plus prize draw, and control

Table 4 Summary of costs

\begin{tabular}{lccccc}
\hline Method & Fixed cost $(\$)$ & Unit cost $(\$)$ & Quantity & Variable cost $(\$)$ & Total cost $(\$)$ \\
\hline LT & 200 & $1.20^{*}$ & 300 & 360 & 560 \\
Prize draw & 200 & 300 & 1 & 300 & 500 \\
LT + prize draw & 200 & 300 & 1 & 300 & 500 \\
& & $1.20^{*}$ & 300 & 360 & 360 \\
Control & 200 & - & 300 & 0 & 200 \\
Total & & & & & 2120 \\
\hline
\end{tabular}

* Unit cost includes the cost of the luggage tag and mailing the tag to the respondents 
groups. Fixed costs included designing the survey. Variable costs included the costs of the luggage tags and the PDA. The control group cost the least at $\$ 200$, which is the design cost for the online survey. The most expensive method, as expected, was the combination of luggage tag and prize draw group ( $\$ 860)$. The cost for the prize draw group was $\$ 500$, while it was $\$ 360$ for the luggage tag group.

\section{Conclusions and recommendations}

Although researchers use internet-based surveys more often than ever, there has been little research on the effect of incentives on the response rate, speed and cost of such surveys. This study attempted to fill in some of the blanks by comparing with each other the different incentives offered to the respondents of web-based surveys. The results indicate that offering a luggage tag to each respondent and including them in a prize draw for a PDA yields the highest response rate. The next best method in terms of response rate was giving a small incentive, such as a key chain, luggage tag or mini-calculator to each respondent. There was no significant difference between including the respondents in a prize draw and offering nothing at all. In terms of response speed, there are no significant differences between each incentive group. The most expensive group was the combination of luggage tag and prize draw.

Based on the findings of this study, it may be recommended that researchers use incentives when conducting online surveys to achieve higher response rates. Further, researchers may do well by not only offering a small prize to all respondents but also entering them into a draw for a bigger prize. However, since offering a prize draw has a minimal effect on the respondents, it is recommended that researchers offer an incentive to each respondent if the combination of a small incentive prize and draw for a bigger prize is impractical due to budgetary considerations. It is also recommended that researchers distribute the promised incentives promptly. It may be a good idea to include a detailed timeline either in the covering email or introductory section of the questionnaire and conditions about how and when the incentives will be distributed. Finally, the authors suggest that the prize draw be handled by an independent agency, such as the dean of a college or a public notary . This may increase respondents' confidence that the prize will actually be distributed and the selection will be made fairly. Finally, researchers may need to use uniform mixed-design in which not only the internet-based methods are used but also other methods, such as mail and fax, are used for parts of the population that 
do not have internet access, in order to achieve the highest and most comprehensive response rate.

\section{Ethical considerations}

When planning, designing, and conducting a research study, the researcher needs to be careful in areas where there may be ethical decisions to be made. In addition, researchers should try to prevent the respondents from behaving subjectively. In this study, the authors suggest that the use of incentives in a web survey increased the response rate significantly. However, researchers need to be aware of the ethical aspects of using incentives in web surveys, of which the main points are listed below.

- Researchers need to distribute the promised incentives promptly as a requirement of honesty. In studies of this kind, one of the biggest ethical problems is not fulfilling the promises made before the survey was distributed to respondents.

- Researchers may need to create environments where every respondent has an equal chance of winning the prize in the case of a prize draw. In addition, the involvement of an objective party such as a public notary for selecting the winner may increase the level of trust that the respondents have in the incentive promises.

- The conditions of the incentives need to be communicated to the respondents openly. The deadlines for the inclusions in the drawings need to be posted in advance.

- The specification of the incentive is important. Researchers need to make sure that the incentive is not something that may affect the responses in any way. Incentives need to encourage but not affect the responses. For example, in a market research survey that investigates the most known shampoo, it is not a good idea to give out samples of a certain branded shampoo as an incentive as this may bias the survey results.

- The incentives should also not be so valuable in price that respondents answer the survey merely to stand a chance of winning the prize. If this is the case, the results may be biased. Therefore, the selection of the incentives needs to be viewed as a serious step of the research process. 


\section{Directions for future research}

In conclusion, internet-based surveys may soon replace the traditional survey methods of data collection. Therefore, researchers would do well to conduct more studies on internet-based survey methodology. Future research may include studies that investigate the format of the web survey's effect on response quality. Similarly, other studies should be conducted by using different incentives and populations.

\section{References}

Albaum, G.S., Evangelista, F. \& Medina, N. (1998) Role of response behaviour theory in survey research: a cross-national study, Journal of Business Research, 42, pp. $115-125$.

Armstrong, J.S. (1975) Monetary incentives in mail surveys, Public Opinion Quarterly, 39, pp. 111-116.

Brennan, M. (1992) The effect of a monetary incentive on mail survey response rates - new data, Journal of the Market Research Society, 34, 2, pp. 173-177.

Cahalan, D. (1951) Effectiveness of mail questionnaire technique in the army, Public Opinion Quarterly, 15, pp. 575-580.

Church, A.H. (1993) Estimating the effects of incentives on mail response rates: a meta-analysis, Public Opinion Quarterly, 57, pp. 62-79.

Cobanoglu, C. (2001) Analysis of Business Travelers' Hotel Selection and Satisfaction. Unpublished thesis. Oklahoma State University, Stillwater OK.

Cobanoglu, N. (1998). Etik-Deontoloji-Hukuk Iliskisi (Ethics-Deontology-Law Relationship), Ankara Saglik Mudurlugu Dergisi, 1, 1, pp. 23-24.

Cobanoglu, N. \& Unsal, S. (1998) Etik ve Deontoloji acisindan AIDS/HIV (AIDS/HIV from Ethical and Deontological Perspectives) Guncel Bilgiler Isiginda AIDS/HIV. Ankara: Turk Eczacilari Birligi Yayini.

Cobanoglu, C., Warde, B. \& Moreo, P. (2001) A comparison of mail, fax, and webbased survey methods, International Journal of Market Research, 43, 4, pp. 441-452.

Collins, R.L., Ellickson, P.L., Hays, R.D. \& McCaffrey, D.F. (2000) Effects of incentive size and timing on response rates to a follow-up wave of a longitudinal mailed survey, Evaluation Review, 24, 4, pp. 347-363.

de Leeuw, E.D. (1992) Data Quality in Mail, Telephone, and Face-to-Face Surveys. Amsterdam: TT Publications.

Dillman, D.A. (1978) Mail and Telephone Surveys: The Total Design Method. New York: Wiley-Interscience.

Dillman, D.A. (1999) Mail and Internet Surveys: The Tailored Design Method (2nd edn). New York: Wiley.

Groves, R.M. \& Couper, M.P. (1998) Nonresponse in Household Interview Surveys. New York: Wiley.

Halpern, S.D., Ubel, P.A., Berlin, J.A. \& Asch, D.A. (2002) Randomized trial of \$5 versus $\$ 10$ monetary incentives, envelope size, and candy to increase physician response rates to mailed questionnaires, Medical Care, 40, 9, pp. 834-839. 
Helgeson, J.G., Voss, K.E. \& Terpening, W.D. (2002) Determinants of mail-survey design factors and respondent factors, Psychology \& Marketing, 19, 3, pp. 303-328.

Huck, S.W. \& Gleason, E.M. (1974) Using monetary inducements to increase response rates from mail surveys, Journal of Applied Psychology, 59, pp. 222-225.

Internet User Numbers in the World (2002) Available at: http://ww91.tiki.ne.jp/ sisyphe/ref/internet_world.htm

Kempthorne, O. (1952) The Design and Analysis of Experiments, pp. 156-157, New York: Wiley.

Ray, N., Griggs, K. \& Tabor, S. (2001) Web Based Survey Research Workshop, WDSI. Available at: http://telecomm.boisestate.edu/research.

Roztocki, N. (2001) Using internet-based surveys for academic research: opportunities and problems. Proceedings of the 2001 American Society of Engineering Management (ASEM) National Conference, Huntsville, AL, pp. 290-295.

Schwarz, N., Hippler, H.J. \& Noelle-Neumann, E. (1992) A cognitive model of response-order effects in survey measurement. In N. Schwarz \& S. Sudman (eds), Context Effects in Social and Psychological Research, pp. 187-199, New York: Springer-Verlag.

Shank, M.D., Darr, B.D. \& Werner, T.C. (1990) Increasing mail survey response rates: investigating the perceived value of cash versus non-cash incentives, Applied Marketing Research, 30, 3, pp. 28-32.

Shaw, M.J., Beebe, T.J., Jensen, H.L. \& Adlis, S.A. (2001) The use of monetary incentives in a community survey: impact on response rates, date quality, and cost, Health Services Research, 35, 6, pp. 1339-1346.

Sheehan, B.K. \& McMillan, J.S. (1999) Response variation in email surveys: an exploration, Journal of Advertising, 39, 4, pp. 45-54.

Vandermeer, B. (2000) Incentive Effect on Response Rates for the 1997 Survey of Household Spending. Statistics Canada: Ministry of Industry.

Wang, P.S., Beck, A.L., McKenas, D.K., Meneades, L.M., Pronk, N.P., Saylor, J.S., Simon, G.E., Walters, E.E. \& Kessler, R.C. (2002) Effects of efforts to increase response rates on a workplace chronic condition screening survey, Medical Care, 40, 9, pp. 752-760.

Zhang, Y. (2000) Using the Internet for survey research: a case study, Journal of the American Society for Information Science, 5, 1, pp. 57-68. 